\title{
Personalized Educational Resources Active Service Implementation Based on Higher-order Singular Value Decomposition Algorithm
}

\author{
https://doi.org/10.3991/ijet.v13i11.9586 \\ Jiansheng Liang \\ Dongguan Polytechnic, Guangdong, China \\ jianshengliang29103@163.com
}

\begin{abstract}
In order to solve the problem of lack of effective active personalized education resources active service mode for online education, massive educational resources have led to the problem of information overload and learning confusion, resulting in a situation with many resources and difficulties in obtaining information, it has been researched on active service system based on educational resources. Firstly, the related theoretical basis for online education resources is elaborated. Then, the existing problems are analyzed and sorted out. Finally, the measures for high-order singular value decomposition algorithm are proposed. The practical application proves that the higher-order singular value decomposition algorithm can effectively realize the personalized educational resource active service and provide help for related scholars in learning resources.
\end{abstract}

Keywords-educational resources, personalized service, high-order singular decomposition algorithm

\section{Introduction}

With the advancement of the national education information resources co-construction and sharing policy, the number and types of educational resources are unprecedentedly abundant, and the cognitive ability of human beings is far behind the speed of information diffusion. The rich teaching resources expand the form of learning and bring the gospel to ubiquitous learning and lifelong learning. However, the massive educational information resources cause cognitive overload, information vagrancy and anxiety problems. Learners' access to personalized learning resources is like finding a needle in the sea, learning from time to time often evolves into an all-encompassing search. The change of technology to education is to transform the passive, mechanical, and "low-input" shallow learning into active, flexible, and "high-input" deep learning. The reality is that learners are lost in the information sea, waste a lot of time and energy to search and select information, and it is difficult to conduct high-quality, in-depth study. Obviously, the rapid growth of educational information resources and the contradiction between people's access to personalized information resources have caused 
new education unfairness. How to reduce the information search cost and the annoyance cost of learners, so that learners with different information literacy can obtain the information resources that suit their needs. It is an inescapable reality to provide educational resources services for learners of different knowledge structures and types of intelligence to meet their individual development needs.

The core of smart education is to integrate education theory and information technology, build a smart learning environment, and provide learners with a good learning experience and customized services tailored to their needs. Wisdom education can also be connected to other systems such as smart cities, using high-order singular value decomposition and other technologies to customize various services for citizens. China's education informatization is experiencing the first phase of hardware platform construction, and the second phase of resource construction is moving towards the integration and innovation stage of technology and education. The current resource allocation model shifts from "concentrated construction, co-construction and sharing" to "service level" and "distributed on-demand push", reflecting the shift from the comprehensive unified service to the personalized delivery service. The allocation of educational resources has gradually shifted from the expansion of content to the improvement of service quality, from the construction of quality education information resources to the accurate placement of personalized educational information resources.

The application of big data technology is bound to promote the development of personalized education. Standardized learning content is more accomplished through student self-learning, while schools and teachers are more concerned with the individualized training of students. Big data will inject new potential into ubiquitous learning, crack the "information overload" deadlock, and provide learners with timely learning support and services, thus providing new opportunities for personalized education.

\section{$2 \quad$ Literature review}

In the study, Kumar et al. (2014) proposed that the learner model is the structured representation and storage of learners' preferences, cognition, ability, and situation, so that researchers can analyze the learning needs of learners. Constructing a suitable learner model is the basis for realizing personalized education resource service and is one of the core parts of the whole service system. It is of strategic significance to improve the active service efficiency of educational resources [1]. O'shea et al. (2014) pointed out in the study that the learner model can be divided from different perspectives: the learner model can be divided into a long-term model and a short-term model according to the stability of the learner's information needs; according to the scope of application of the model, it can be divided into a general model and a specific model; according to the learner's participation in system modeling, the student model construction process can be divided into three ways of explicit, implicit, the combination of explicit and implicit [2]. Li et al. (2014) explored and constructed a student model based on learner's cognitive style from the network data, and divided the students into four types: speech, representation, overall, and analysis [3]. Cook et al. (2015) adopted the questionnaire survey method, and the Gunawardena interactive knowledge construction 
hierarchical model was used as the coding basis, and the content analysis method was used to conduct empirical research. The results showed that personality traits such as self-efficacy, learning motivation, achievement attribution and learning style were related to learners' knowledge construction level [4]. Wu et al. (2015) pointed out in the research that from the perspective of network technology, the information pull mode adopts a point-to-point transmission method. When multiple users need one piece of information, network congestion may occur, the time for users to obtain information may be prolonged, and the efficiency of obtaining information by the user may be reduced. In addition, because the server is not actively but passively service to the user, it leads to poor timeliness of information acquisition for user. The user in the information pull mode must actively send out an information request and search for information on the server, which is a typical way of obtaining information for "people looking for information" [5]. Cain et al. (2015) pointed out that tensors were first used in the fields of psychometry, quantum physics, and quantum chemistry. Since the tensor can better represent the internal structure of high-dimensional data, it can achieve better results than the traditional method. In recent years, tensor methods have been successfully applied in the fields of computer vision, signal and image processing, and machine learning. In the era of big data, tensor has become an emerging tool for big data analysis [6]. Ainscough et al. (2016) pointed out that high-order singular value decomposition is a kind of tensor Tucker decomposition, and it is an extension of matrix singular value decomposition and principal component analysis in multidimensional matrix, which has been successfully applied in many fields [7]. In the field of data compression and clustering, Banerjee et al. (2016) used high-order singular value decomposition to transform high-order tensors into low-order spaces and then clustered them in low-order spaces. In the field of personalized web search, the Cube SVD algorithm is proposed, which uses HOSVD to extract data from search engines and improve web search results [8].

\section{$3 \quad$ Methodology}

\subsection{Active service architecture for personalized education resources}

In the context of big data, it is necessary to fully integrate the advantages of human intelligence and technology, establish a personalized educational resource active service system, and resolve the contradiction between the current massive educational resources and personalized service needs. Faced with the complex mechanism of learning, it is not scientific enough to rely entirely on algorithms to recommend learning resources. Therefore, it is necessary to combine the precise recommendation algorithm, the automatic push technology and the intuition and wisdom of people in dealing with complex problems, highlighting the value of human-machine combination. Combine computer technology with knowledge management methods. After accurately understanding and analyzing the information needs of users, make full use of the advantages of the network environment, select appropriate knowledge from the information resource library, and provide knowledge services to information demanders in a timely 
and proactive manner so that appropriate knowledge can be transmitted to the right people at the right time.

The knowledge active service platform includes three key components: knowledge requirement analysis module, knowledge space management module, and knowledge push service module. The knowledge requirement analysis module determines the user's knowledge needs by tracking user network behavior, interpersonal network data and part of the survey data. This module is the premise for personalized push. The key of knowledge needs analysis is to construct a learner interest feature model, pay attention to the user profile to understand its learning situation and track its learning behavior. The knowledge space management module mainly processes, manages, and integrates various forms of resources into an alternative resource list. The premise of knowledge management is to construct a learning resource feature model, and classify, characterize, automatically retrieve, and dynamically reconstruct the learning resources. The knowledge push service module mainly extracts the information resources that have been processed and aggregated and then pushes them to the user in an appropriate form. This research aims to promote the fairness of educational process with information technology and constructs a personalized educational resource active push service system including learner model, resource model and recommendation system, as shown in Figure 1.

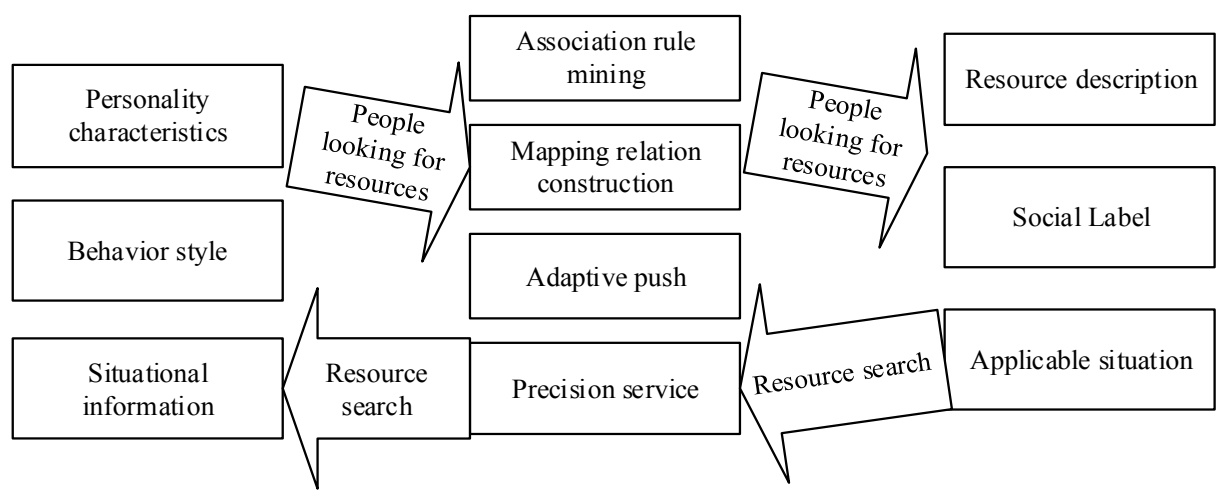

Fig. 1. The structure of individualized educational resources

The personalized education resource active service push system is mainly based on the principle that "people are looking for resources and resources find people". By establishing a matching mapping relationship between learners and educational resources, it promotes adaptive educational resources for learners, finds suitable learners for educational resources, and realizes on-demand push and precise services for educational resources. In the learner module, the learner's personality characteristics, behavioral style, and context information are integrated into a learner model, which is passed to the recommendation engine to analyze and predict the learner's needs, so as to achieve the purpose of people looking for resources; in the education resource module, it focuses on the integration of educational resources metadata description, user social annotation and applicable scenarios to construct an educational resource model, which is 
then passed to the recommendation engine to predict the adaptation of educational resources to achieve the purpose of resources to find people; in the recommendation engine module, it mainly performs correlation analysis and association rule mining on the information in passed learner model and the information in the educational resource model. A matching mapping relationship is established between learners and educational resources, so as to recommend appropriate educational resources for learners and recommend suitable learners for educational resources according to the rules of adaptive push and the concept of precise service.

\subsection{Learner model of personalized education resources}

The essence of educational resource active service is to establish a matching and mapping relationship between the learner and the learning resource, and the similarity between the learner feature vector and the learning resource feature vector needs to be calculated. It ensures that quality resources (including information resources, human resources, learning strategies and methods) can actively serve learners. Learner characteristics are modeled as comprehensively and stereoscopically as possible with as few feature attributes as possible. In order to explore the factors affecting learners' choice of resources and their internal relevance structure, the interpretative structural model method is used to analyze the factors affecting learners' choice of resources and establish a learner model that affects learners' choice of resources.

Table 1. Factors affecting learners' resource selection

\begin{tabular}{|c|c|}
\hline Presenter & Influence factor \\
\hline IMS (2001) & $\begin{array}{l}\text { Personal information, language, abilities, preferences, } \\
\text { knowledge, skills, and transcripts. }\end{array}$ \\
\hline IEEE (2002) & $\begin{array}{l}\text { Personal information, learner preferences, documents, } \\
\text { achievements, security, learning relations. }\end{array}$ \\
\hline $\begin{array}{l}\text { China Education Information Committee } \\
\text { of the Ministry of Education (2004) }\end{array}$ & $\begin{array}{l}\text { Personal information, academic information, management in- } \\
\text { formation, relationship security, and works. }\end{array}$ \\
\hline Jiang qiang (2012) & Learning style, cognitive level, learning behavior \\
\hline Zhang wen lan (2015) & $\begin{array}{l}\text { Media preferences, cognitive preferences, learning strategies, } \\
\text { attitudes, motivations, attention, reasons. }\end{array}$ \\
\hline Zhang jian ping (2010) & Knowledge base, cognitive ability, learning style. \\
\hline Li wei shi (2011) & $\begin{array}{l}\text { Knowledge model, interest manipulation, retrieval operation, } \\
\text { collection operation, learning behavior. }\end{array}$ \\
\hline Lv yan hua (2010) & Interest, information acquisition motivation, behavior ability. \\
\hline
\end{tabular}

In order to construct the learner model, it is necessary to extract the characteristic factors that affect the learner's resource selection. The literatures related to the learner model at home and abroad are sorted and summarized, and the characteristic factors that influence the learners' choice of resources are formed as shown in Table 1. It is not difficult to find that the learner model has many attribute factors, including personal basic information, interest preferences, knowledge base, learning style, cognitive ability, learning objectives, learning behavior, portfolio, and relationship information. On 
the surface, these factors are complicated, but through further sorting and induction, it can be basically summarized as: basic information of individuals, knowledge base, cognitive ability, learning situation information, and learning behavior information.

According to the hierarchical division of each influencing factor, the interpretation structural model (ISM) diagram shown in Figure 2 is drawn, which is a 6-level directed hierarchical structure model. The higher the level, the bigger the coverage it covers; the lower the level, the smaller the coverage it covers. The bottom-up arrow indicates that the lower level will directly affect the high level.

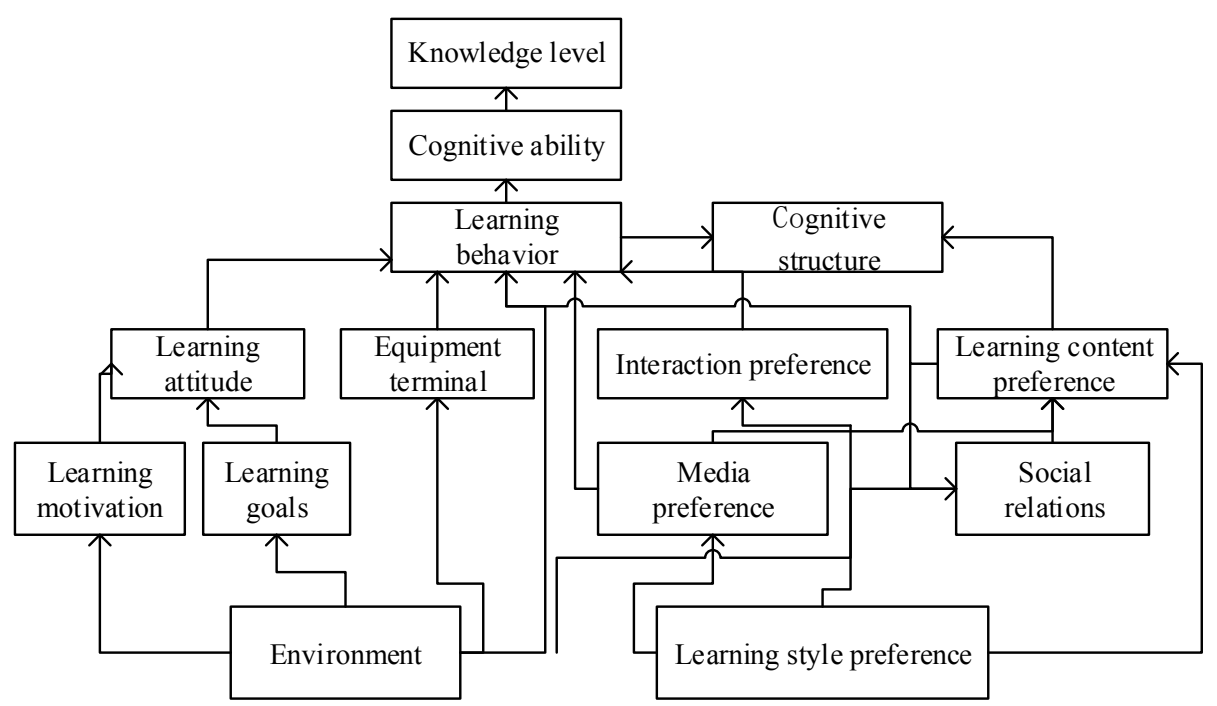

Fig. 2. Learner structure model ISM

\subsection{Application of high-order singular value decomposition algorithm}

Roughly speaking, an N-order tensor is an object consisting of $\mathrm{N}$ parameters. The dimension of the tensor refers to the modulus of the tensor. Vectors and matrices can be thought of as first- and second-order tensors, respectively. In this article, the thirdorder tensor is applied. Therefore, for the convenience of description, some of the theoretical discussions in this section are only for third-order tensors.

The tensor decomposition is generally divided into CP decomposition and Tucker decomposition, where Tucker decomposition is a form in which a tensor is decomposed into a core tensor multiplied by a factor matrix on each module. High-order singular value decomposition (HOSVD) is a kind of Tucker decomposition, which is an extension of matrix singular value decomposition (SVD) to higher order. The SVD is introduced below. Singular value decomposition (SVD) is an important matrix decomposition method. It can decompose a complex matrix into several small sub-matrices. These sub-matrices can reflect the main features of the original matrix to some extent.

Singular value decomposition (SVD). SVD decomposes the matrix into the following form: 


$$
\mathrm{A}=\mathrm{U} \sum \mathrm{V}^{\mathrm{T}}
$$

From the singular value decomposition results, because the size of the singular value can be understood as the importance of the corresponding feature, the smaller singular value and its corresponding feature (often some noise data) can be removed in the approximate representation, thus retaining the most important features to achieve data cleaning and data dimensionality reduction.

High-order singular value decomposition (HOSVD). HOSVD is a form of decom-

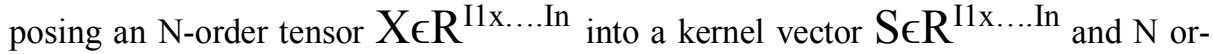
thogonal matrix models:

$$
\underline{\mathrm{X}} \approx \underline{\mathrm{S}} \mathrm{X}_{1} \mathrm{U}^{(1)} \mathrm{X}_{2} \mathrm{U}^{(2)} \ldots \mathrm{X}_{\mathrm{N}} \mathrm{U}^{(\mathrm{N})} .
$$

It can be concluded that HOSVD is a kind of tensor Tucker decomposition, which requires that all factor matrices in Tucker decomposition are orthogonal and the nuclear tensor is fully orthogonal.

The nuclear tensor S must satisfy the following two conditions: (1) full orthogonality: the slices on each mode are orthogonal to each other. (2) pseudo-diagonality: the Frobenius norm of the slice on each mode decreases as the coordinates increase, where the norm is similar to the singular value in the matrix SVD decomposition.

Unfolding refers to the process of rearranging all elements in a tensor into a matrix in a certain order. This is also the process of tensor matrixing. In general, the mode-n expansion of the tensor is performed by arranging the column vectors in the tensor mode-n direction into elements in the matrix original tensor A. The row coordinates mapped to the elements in the matrix (n) A are the same as the coordinates on the original tensor mode-n, and the column coordinates are combinations of the coordinates on the modulo other than mode-n. That is:

$$
j=1+\sum_{\substack{K=1 \\ K \neq n}}^{N}\left(i_{k}-1\right) . J_{k}, J=\prod_{\substack{m=1 \\ m \neq n}}^{K-1} I_{m} .
$$

In all kinds of multiplications of tensors, the most commonly used ones are single modular multiplication. "Single mode" means that the tensor only uses the column vector on first modulo (order) to participate in the calculation. For example, for an $\mathrm{N}$-order tensor and a vector's single-modulus multiplication, there are $\mathrm{N}$ single-mode multiplication options. The tensor single modulus multiplication can be roughly divided into tensor and vector single modulus multiplication, tensor and matrix single modulus multiplication, tensor and tensor single modulus multiplication.

In the compression of matrix data, low rank approximations are often used: for the case where the modulus length satisfying the orthogonality is constant, the k-order rank approximation optimal for a given matrix F can be realized by SVD.

The solution process of high-order singular value decomposition generally includes the following steps: firstly, the original tensor is expanded into a matrix according to each dimension, and a matrix is subjected to singular value decomposition. According to the size of the singular value after decomposition, the left singular matrix is truncated 
to obtain a factor matrix in each dimension (representing the main characteristic component of the data in the dimension); secondly, the original tensor and each factor matrix are subjected to modular multiplication in the corresponding dimension to obtain a core tensor G; finally, the core tensor $\mathrm{G}$ and the factor matrix $\mathrm{U}$ in each dimension are modularly multiplied in the corresponding dimensions to obtain an approximate tensor $\mathrm{X}$.

\subsection{Realization of personalized education resources active services}

The architecture of the active push service of educational resources mainly includes the database layer, the technical operation layer, and the service layer. In order to realize the overall planning of personalized education resources active service, it is mainly based on learner's learning behavior record and learning situation. Through tensorbased high-order singular value decomposition algorithm, clustering and recommendation are realized, which can effectively realize active push based on dynamic situation and learning state.

The concrete construction steps of the tensor model are as follows:

The first step is to construct a subscript for each sub-tensor. In order to express and reflect the main information of the learner, the main attributes are usually extracted for analysis. To this end, the student number, gender, time, cognitive style, media preference, information processing, and learning persistence are selected as the representation dimension of the learner's tensor. Then the learner tensor subscript tuple is constructed, such as tuple $(1,2,1,2,3,1,2)$, indicating the information of learner No. 1, gender is No. 2 (female), in the time period 1 (in class), its cognitive style belongs to category 2 (field independent), media preference belongs to category 3 (audio), information processing method is category 1 (abstract-random), and learning persistence is category 2 (low persistence). Similarly, the resource number, media type, interaction type, learning goal, and difficulty level are selected as the representation dimension of the resource tensor and the subscript tuple of the resource tensor is constructed. In addition, in order to represent the dimension of the learning record tensor, several attributes such as the learner's student number, the learning resource number, the learning time, the learning place, the device, and the learning duration are selected, and the learning record subscript is constructed.

The second step: the corresponding sub-tensor is established according to subscript for each sub-tensor. By designing the Tensor-sub (Sub) function, the sub-tensor subscript tuple is converted into the corresponding sub-tensor.

The third step: the learner sub-tensor, the resource sub-tensor and the learning record sub-tensor are merged. The learner sub-tensor and the learning record sub-tensor are merged by the Join operation (student number, time period), and then merged with the resource tensor (resource number) to obtain the final "learner-resource" fusion tensor model T. 


\subsection{Personalized education resource recommendation based on high-order singular value decomposition}

The traditional recommendation system evolved from a two-dimensional model $<$ user, resource > to a three-dimensional model < user, label, resource>, and its recommendation algorithm includes a content-based recommendation algorithm and a collaborative filtering recommendation algorithm, which are all operated on a matrix of lowdimensional space. For the three-dimensional model, the three-dimensional relationship is generally split into two two-dimensional models $<$ user, label $>$ and $<$ resource, label $>$ for analysis and recommendation. This method of splitting loses the characteristic structure and interrelationship between each other, because the traditional matrix can only represent the relationship between the two. The tensor model can guarantee the information integrity of three-dimensional and even higher-dimensional space and the complex relationship between expression data, which has certain advantages. The tensor-based high-order singular value decomposition can also maintain the representation structure information of high-dimensional space completely, and it explores the potential semantic association between data from multiple dimensions to achieve more accurate recommendation.

There is a large amount of noise in the tensor data, so the noise data must be effectively cleaned before the recommended operation. The tensor-based high-order singular value decomposition firstly expands the original tensor into a corresponding matrix according to each dimension and performs SVD decomposition on each matrix. Then, according to the actual needs and the importance of the singular value after decomposition, the factor matrix of each dimension is truncated, and only the most important data features are retained. Therefore, high-order singular value decomposition is an effective method for cleaning noise data, and it can also perform synchronous dimensionality reduction on the original tensor in each dimension.

The specific recommendation process based on tensor high-order singular value decomposition is as follows:

The first step: the original tensor is subjected to high-order singular value decomposition. The tensor decomposition is performed with an alternating least squares (ALS). In the decomposition process, the SVD decomposition of the matrix of the original tensor expanded in each dimension is required, and the truncation is performed according to the required singular value.

The second step: the approximate tensor is reconstructed. After truncation, the nuclear tensor represents the importance of different features in different dimensions. The truncation operation of high-order singular value decomposition can retain the most important features in each dimension. The original tensor can be reconstructed by the modulus multiplication of the kernel tensor and the matrix of the factors to obtain an approximate tensor.

The third step: the recommendation list Top-k is generated. The reconstructed approximate tensor not only removes the noise data, but also retains the important features of each dimension, and also turns the original zero element into a non-zero element according to the association of other known data, representing the possibility that a learner accesses a certain resource at a specified time, place, and device. 


\section{$4 \quad$ Results}

Birds of a feather flock together. Learners with the same learning style often choose similar learning resources. People with the same personality and hobbies tend to prefer similar learning behaviors. Therefore, they will have similar learning records and learning trajectories. For the historical learning trajectory data, through the high-order singular value decomposition of the learner-resource fusion tensor, the association analysis can be integrated from multiple dimensions to achieve clustering between learners and resources in high-dimensional space, so as to recommend a learning community for target learners.

The high-order singular value decomposition algorithm can not only reflect the clustering idea of principal component analysis, but also reflect the recommendation strategy based on collaborative filtering. By clustering the learners, it is possible to recommend similar learners to the designated target learners, thereby helping the learners to form a learning community and generate good learning interactions and cooperative learning. Based on the learner's learning behavior record, it is possible to recommend suitable resources to the required learners and promote the rational use of resources. Therefore, inspired by the five laws of library science proposed by Ranganathan, the significance of the educational resource recommendation system is summarized. Firstly, the education resource recommendation system enables learning resources to be delivered to learners in a convenient, efficient, accurate, and timely manner; secondly, it can find a learning resource for each learner that suits their needs; thirdly, it can find a suitable learner for each resource; fourthly, the education resource recommendation system can reduce the time cost and energy cost for learners to obtain learning resources; fifthly, many learners and resources constitute an organic ecosystem. Similar learners can form a learning community and explore knowledge in collaboration. The value of each resource can be tapped, and each learner's interest in learning can be protected.

\section{Conclusion}

The implementation of educational resource active push service: in the traditional collaborative filtering recommendation system and content-based recommendation system, most of them are information recommendation based on two-dimensional model. This recommendation covers three dimensions of learners, learning records, and resources. Although the traditional algorithm can be changed from two-dimensional to three-dimensional, many feature details may be lost during the conversion process. In terms of the richness of the learner's characteristics and the complexity of the learning process, the tensor can not only fully represent the characteristic information of the high dimensional data, but also has a strong explanatory effect on the representation method. Therefore, tensors are used to represent learner characteristics, behavioral records, learning contexts and learning processes. However, high-dimensional tensor and extremely sparse data may lead to waste of storage units and excessive time complexity 
of calculations. The tensor not only has multi-dimensional sparse matrix storage features, but the tensor-based high-order singular value decomposition algorithm can realize the effective correlation and processing of the high dimensional data, which is very suitable for educational resource recommendation.

The tensor-based high-order singular value decomposition algorithm provides an integrated solution for clustering and recommendation. The algorithm embodies the clustering idea of principal component analysis and the recommendation strategy based on collaborative filtering. The clustering of learners helps learners form a learning community and produce good learning interactions and collaborative learning. Based on the recommendation of the student's learning behavior record, the resource is dynamically served to the learner. Through the recommendation of educational resources, the learning resources are promptly and accurately delivered to the learners, reducing the time cost and energy cost of the learners to obtain learning resources; it enables each learner to find the learning resources that suit their needs, and it makes each resource find the learner that suits it; students and resources constitute an ecological system of harmonious symbiosis. Students learn and explore knowledge together according to their interests. The value of resources is fully excavated, and resources are recommended to the most needed learners.

\section{Reference}

[1] Kumar, H., Lee, S., Kim, H. G. (2014). Exploiting social bookmarking services to build clustered user interest profile for personalized search. Information Scienc-es, 281: 399-417. https://doi.org/10.1016/j.ins.2014.05.008

[2] O'Shea, J., Kanno, Y., Chan, A. (2014). In search of magic bullets: the golden age of immunotherapeutics. Cell, 157(1): 227-40. https://doi.org/10.1016/j.cell.2014.03.010

[3] Li, Y. M., Chou, C. L., Lin, L. F. (2014). A social recommender mechanism for lo-cationbased group commerce.Information Sciences, 274(8): 125-142. https://doi.org/10.1016/ j.ins.2014.02.079

[4] Cook, D., Swinton, M., Toledo, F., Clarke, F., Rose, T., Handbreckenridge, T. (2015). Personalizing death in the intensive care unit: the 3 wishes project: a mixed-methods study. Annals of Internal Medicine, 163(4): 271-9. https://doi.org/10.7326/M15-0502

[5] Wu, X., Chen, H., Zhang, Q., Zhang, S., Wu, G., Liu, J. (2015). Knowledge engi-neering with big data. IEEE Intelligent Systems, 30(5): 46-55. https://doi.org/10.1109/MIS.2015.56

[6] Cain, J., Piascik, P. (2015). Are serious games a good strategy for pharmacy educa-tion. American Journal of Pharmaceutical Education, 79(4): 47. https://doi.org/10.5688/ajpe7 $\underline{9447}$

[7] Ainscough, B. J., Griffith, M., Coffman, A. C., Wagner, A. H., Kunisaki, J., Choudhary, M. N. (2016). Docm: a database of curated mutations in cancer. Na-ture Methods, 13(10): 806. https://doi.org/10.1038/nmeth.4000

[8] Banerjee, S., Mitra, S., Shankar, B. U. (2016). Single seed delineation of brain tu-mor using multi-thresholding. Information Sciences, 330(C): 88-103. https://doi.org/10.1016/j.ins. $\underline{2015.10 .018}$ 
Paper-Personalized Educational Resources Active Service Implementation Based on Higher-order Sing...

\section{$7 \quad$ Author}

Jiansheng Liang is with Dongguan Polytechnic, Guangdong 523808, China (jianshengliang29103@163.com).

Article submitted 24 September 2018. Resubmitted 18 October 2018. Final acceptance 02 November 2018. Final version published as submitted by the author. 